\title{
Recent progress toward high performance above the Greenwald density limit in impurity seeded discharges in limiter and divertor tokamaks*
}

\author{
J. Ongena, $\left.{ }^{\dagger}, 1, a\right)$ R. Budny, ${ }^{2}$ P. Dumortier, ${ }^{1}$ G. L. Jackson, ${ }^{3}$ H. Kubo, ${ }^{4}$ A. M. Messiaen, ${ }^{1}$ \\ M. Murakami, ${ }^{5,3}$ J. D. Strachan, ${ }^{2}$ R. Sydora, ${ }^{6}$ M. Tokar, ${ }^{7}$ B. Unterberg, ${ }^{7}$ U. Samm, ${ }^{7}$ \\ P. E. Vandenplas, ${ }^{1}$ R. Weynants, ${ }^{1}$ N. Asakura, ${ }^{4}$ M. Brix,${ }^{7}$ M. Charlet,${ }^{8}$ I. Coffey,${ }^{8}$ \\ G. Cordey, ${ }^{8}$ S. K. Erents, ${ }^{8}$ G. Fuchs, ${ }^{7}$ M. von Hellermann, ${ }^{9}$ D. L. Hillis, ${ }^{5}$ J. Hogan, ${ }^{5}$ \\ L. D. Horton, ${ }^{10}$ L. C. Ingesson, ${ }^{9}$ K. Itami, ${ }^{4}$ S. Jachmich, ${ }^{1}$ A. Kallenbach, ${ }^{10}$ H. R. Koslowski, ${ }^{7}$ \\ A. Kraemer-Flecken, ${ }^{7}$ K. D. Lawson, ${ }^{8}$ A. Loarte, ${ }^{11}$ G. P. Maddison, ${ }^{8}$ G. Mank, ${ }^{7}$ \\ G. R. McKee,${ }^{3,12}$ A. Meigs, ${ }^{8}$ F. Milani, ${ }^{8}$ P. Monier-Garbet, ${ }^{13}$ M. F. F. Nave ${ }^{14}$ M. E. Puiatti, ${ }^{15}$ \\ V. Parail, ${ }^{8}$ J. Rapp, ${ }^{7}$ S. Sakurai, ${ }^{4}$ S. Sharapov,${ }^{8}$ F. Sartori, ${ }^{8}$ M. Stamp, ${ }^{8}$ H. Tamai, ${ }^{4}$ \\ G. Telesca, ${ }^{15} \mathrm{M}$. Valisa, ${ }^{15} \mathrm{G}$. Van Wassenhove, ${ }^{1}$ B. Weyssow, ${ }^{16} \mathrm{~K} .-\mathrm{D}$. Zastrow, ${ }^{8}$ \\ and EFDA-JET workprogramme contributors \\ ${ }^{1}$ LPP/ERM-KMS, Association EURATOM-Belgian State, B-1000 Brussels, Belgium \\ ${ }^{2}$ Princeton Plasma Physics Laboratory, Princeton University, New Jersey 08543 \\ ${ }^{3}$ DIII-D National Fusion Facility, San Diego, California 92186-5698 \\ ${ }^{4}$ Japan Atomic Energy Research Institute, Naka Fusion Research Establishment, Nakamachi, Naka-gun, \\ Ibaraki-ken 311-0913, Japan \\ ${ }^{5}$ Oak Ridge National Laboratory, Oak Ridge, Tennessee 37831 \\ ${ }^{6}$ University of Alberta, Edmonton, Canada \\ ${ }^{7}$ IPP, Forschungszentrum Jülich GmbH, EURATOM Association, D-52425 Jülich, Germany \\ ${ }^{8}$ EURATOM/UKAEA Fusion Association, Culham, United Kingdom \\ ${ }^{9}$ FOM-IVP, EURATOM Association, Postbus 1207, NL-3430 BE Nieuwegein, Netherlands \\ ${ }^{10}$ Max-Planck IPP, EURATOM Association, D-85748 Garching, Germany \\ ${ }^{11}$ EFDA-CSU, D-85748 Garching, Germany \\ ${ }^{12}$ University of Wisconsin-Madison, Madison, Wisconsin \\ ${ }^{13}$ CEA Cadarache, F-13108 St. Paul lez Durance, France \\ ${ }^{14}$ CFN, EURATOM-IST Associação, 1096 Lisbon, Portugal \\ ${ }^{15}$ Consorzio RFX, Corso Stati Uniti 4, 35127 Padova, Italy \\ ${ }^{16}$ Université Libre de Bruxelles, Association "EURATOM-Belgian State", Physique Théorique et \\ Mathématique, Unité de Physique des Plasmas, 1050 Brussels
}

(Received 27 October 2000; accepted 16 February 2001)

\begin{abstract}
An overview is given of recent advances toward the realization of high density, high confinement plasmas with radiating mantles in limiter and divertor tokamaks worldwide. Radiatively improved mode discharges on the Torus Experiment for Technology Oriented Research 94 (TEXTOR-94) [Proceedings of the 16th IEEE Symposium on Fusion Engineering, 1995 (Institute for Electrical and Electronics Engineers, Piscataway, NJ, 1995), p. 470] have recently been obtained at trans-Greenwald densities (up to $\bar{n} / n_{\mathrm{GW}}=1.4$ ) with high confinement mode free of edge localized modes (ELM-free $H$-mode) confinement quality. Experiments in DIII-D [J. Luxon et al., Proceedings of the 11th IAEA Conference on Plasma Physics and Controlled Nuclear Fusion Research (International Atomic Energy Association, Vienna, 1987), Vol. 1, p. 159] divertor plasmas with a low confinement mode edge have confirmed the dramatic changes in confinement observed with impurity seeding on TEXTOR-94. Recent experiment with impurity seeding on the Joint European Torus [Rebut et al., Fusion Eng. Des. 22, 7 (1993)], and the Japanese Atomic Energy Research Institute Tokamak 60 Upgrade [Horiike et al., Fusion Eng. Des. 16, 285 (1991); Hosogane et al., Proceedings of the 16th IAEA Conference on Plasma Physics and Controlled Nuclear Fusion Research Montreal, 1987 (International Atomic Energy Association, Vienna, 1987), Vol. 3, p. 555] have extended high confinement in ELMy $H$-mode plasmas to higher densities. Finally, recent progress in the physical understanding of radiating mantle plasmas is summarized. (C) 2001 American Institute of Physics. [DOI: 10.1063/1.1364513]
\end{abstract}

\section{INTRODUCTION}

An operational regime offering a solution for particle and heat exhaust and at the same time combining high con-

*Paper CI2 4, Bull Am. Phys. Soc. 45, 60 (2000).

Invited speaker.

${ }^{\text {a) }}$ Researcher at NFSR Belgium. finement at high densities close to the empirical Greenwald limit $n_{\mathrm{GW}}$ (Ref. 1) is of crucial importance for a next step fusion device $\left(n_{\mathrm{GW}}\left[10^{20} \mathrm{~m}^{-3}\right]=I_{p}[\mathrm{MA}] /\left(\pi a^{2}\left[\mathrm{~m}^{2}\right]\right)\right.$, where $I_{p}$ is the plasma current and $a$ the minor plasma radius). Such an integrated scenario is offered by the radiatively improved mode (RI mode) of the Torus Experiment for Technology Oriented Research 94 (TEXTOR-94), ${ }^{2,3}$ obtained by seeding 
impurities (Ne, Ar, or $\mathrm{Si})$ in the plasma edge. Impurity seeding not only leads to a radiating plasma edge, but surprisingly to a regime where energy confinement is linearly dependent upon plasma density, resulting in a confinement quality as good as ELM-free $H$-mode (high confinement mode free of edge localized modes) at the Greenwald density. This regime has been demonstrated for quasistationary periods of several seconds, equivalent to more than 160 energy confinement times or several skin times. ${ }^{4}$ In fact, it is very similar to the $Z$ mode $^{5,6}$ of ISX-B (Impurity Study Experiment B) which was, however, observed in a narrower parameter range.

Because of the interesting properties of the RI mode on TEXTOR-94, several tokamaks around the world (for an overview of radiating mantle experiments on a large number of tokamaks before 1998 see Refs. 7-9), including ASDEX-Upgrade ${ }^{10}$ (Axisymmetric Divertor Experiment Upgrade), TFTR $^{11}$ (Tokamak Fusion Test Reactor), DIII-D, ${ }^{12}$ JT-60U ${ }^{13}$ (Japanese Atomic Energy Research Institute Tokamak 60 Upgrade), and JET ${ }^{14-17}$ (Joint European Torus), have undertaken impurity seeding experiments, and several theoretical groups are trying to establish a better understanding of the influence of impurity seeding on the confinement properties of tokamak plasmas. Each tokamak device shows different properties of impurity seeded plasmas, but the common trend is the possibility to achieve high confinement at higher densities approaching or even exceeding the Greenwald limit due to a decreased bulk turbulence and a concomitant increase in particle confinement time. It is the aim of this paper to give an overview of the present worldwide experimental efforts, of the results obtained, and of the theoretical progress in the physical understanding of impurity seeded plasmas.

\section{RECENT RI-MODE RESULTS IN TEXTOR-94}

TEXTOR-94 is a medium size tokamak $(R=1.75 \mathrm{~m}, a$ $=0.46 \mathrm{~m})^{18}$ equipped with a toroidal pumped limiter ALT-II (Advanced Limiter Technology II). ${ }^{19}$ The RI mode in TEXTOR-94 is obtained with (i) neutral beam co-injection (NBI-co) heating possibly combined with NBI-counter and/or ion cyclotron resonance heating (ICRH), (ii) with a feedback controlled seeding of impurities $(\mathrm{Ne}, \mathrm{Ar})$ in a boronized machine, or (iii) sputtering of $\mathrm{Si}$ in a siliconized machine, possibly further enhanced by feedback controlled $\mathrm{Ne}$ seeding. The type of impurity chosen for a given plasma is such that a good mantle radiation has to occur. Confinement in the RI mode is given by $\tau_{\mathrm{RI}}=K \bar{n} P_{\text {tot }}^{-0.66}$ (where $K$ $=0.18$ if the confinement time $\tau_{\mathrm{RI}}$ is expressed in $\mathrm{s}$, the central line averaged electron density $\bar{n}$ in $10^{20} \mathrm{~m}^{-3}$, and the total heating power $P_{\text {tot }}$ in MW). Highest confinement is reached in the absence of gas puffing, when the discharge is fueled predominantly by the particles from the neutral beams and to a lesser extent by the wall. The linear dependence of confinement in the RI mode of TEXTOR-94 is however limited by the experimental limit $\beta_{N} \sim 2$ (accompanied by the appearance of neoclassical tearing modes ${ }^{20}$ ) and confinement rollovers or even backtransitions occur ${ }^{21}$ when conditions close to this limit are reached. Indeed, impurity seeding leads to an increase in the particle confinement time and a reduction in the edge fluctuations, as measured by a reciprocating probe. $^{22}$ This has as a consequence that in many cases (i) TEXTOR-94 RI-mode discharges can maintain a density value close to the Greenwald limit by beam fueling only and (ii) that a small amount of gas puffing can be sufficient to obtain a large density increase, leading to high values for $\tau_{E}$ and consequently also for $\beta_{N}$. Therefore the density increase in this regime is constrained by the maximum $\beta_{N}$. Strong deuterium puffing combined with precautions to avoid MARFE formation (i.e., fresh boronization or siliconization possibly combined with an outward shift of the plasma horizontal position) on the other hand leads to higher densities (up to $\bar{n} / n_{\mathrm{GW}}=2.0$ ), ${ }^{23}$ but confinement is reduced down to $L$-mode level. The degrading property of gas fueling is used to advantage by applying a well-chosen moderate level of gas puffing in the discharge, reducing the high confinement of the RI mode at the highest densities to levels avoiding $\beta$ limitations. The net result is that even higher densities (up to $\left.\bar{n} / n_{\mathrm{GW}}=1.4\right)$ can now be reached in TEXTOR-94 with ELM-free $H$-mode confinement quality.

A statistical overview of the normalized confinement time $\tau_{E} P^{0.66} / I_{p}$ versus the Greenwald factor $\bar{n} / n_{\mathrm{GW}}$ is given in Fig. 1. The data in this diagram belong to a series of shots obtained at $B_{t}=2.25 \mathrm{~T}$ and $I_{p}$ between 350 and $400 \mathrm{kA}$, with $\mathrm{Si}$ as radiating impurity resulting from sputtering of the siliconized wall of the machine. Different symbols indicate different fueling rates. The loci of $\mathrm{L}_{-}, \mathrm{H}$-, and RI-mode confinement and of the limit $\beta_{N}=2$ are also indicated. Note that RI-mode scaling also describes the Neo-Alcator or linear Ohmic confinement of TEXTOR-94. ${ }^{4}$ Points with the lowest fueling rates all follow the RI-mode scaling, which constitutes the best possible confinement that can be achieved in TEXTOR at high density. At higher fueling rates a departure is seen from pure RI-mode confinement, and it is clear that by adapting the fueling one can stay just under the $\beta$ limit and nevertheless follow $H$-mode confinement. The data points belonging to the highest fueling rates all have a confinement close to $L$ mode but can reach higher densities (up to $\bar{n} / n_{\mathrm{GW}}=1.8$ for the discharges considered here).

Confinement improvement is closely linked to turbulence suppression in TEXTOR-94. Theoretical models (see also Sec. VI $)^{24}$ predict a reduction of the ion thermal gradient (ITG) turbulence in the bulk plasma due to the presence of the impurity and the peaking of the density profile. The effect of strong gas puffing is to broaden the density profile, and therefore at the same time to reduce the stabilization of ITG modes. Modeling of this effect ${ }^{25}$ shows the necessity to also increase the diffusion coefficient of the main plasma particles at the plasma edge in the presence of a strong gas puff. This effect could be linked to a destabilization of the resistive ballooning modes in the edge in the presence of a sufficiently large density of neutrals in the edge. ${ }^{26}$ Indeed, reflectometer measurements show an increase in the amplitude of edge density fluctuations $(2 \mathrm{~cm}$ inside the last closed magnetic surface) under RI-mode conditions ${ }^{25}$ with moderate fueling.

The sensitivity of confinement to $\mathrm{D}_{2}$ gas puffing is illustrated in Fig. 2, for two discharges obtained with $\mathrm{Si}$ as radi- 


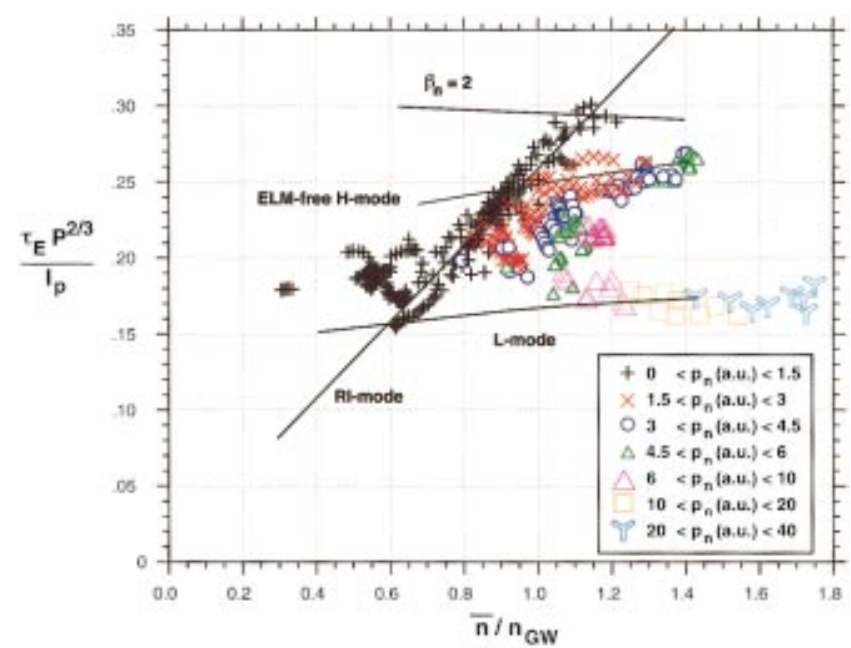

FIG. 1. (Color) Normalized confinement time $\tau_{E} P^{0.67} / I_{p}$ vs Greenwald factor $\bar{n} / n_{\mathrm{GW}}$. Different symbols correspond to different values for the edge neutral pressure $p_{a}$. The lines correspond to the loci of RI mode, $L$ mode, ELM-free $H$ Mode and the limit $\beta_{N}=2$.

ating impurity obtained from sputtering of the siliconized wall of the machine. Additional heating for both discharges consists of $1.35 \mathrm{MW}$ of NBI-co and $750 \mathrm{~kW}$ of ICRH, and is started at, respectively, $t=0.6 \mathrm{~s}$ and $t=0.7 \mathrm{~s}$. A preprogrammed ramp of the density is applied at $t=1.2 \mathrm{~s}$. Confinement characteristics of the plasma are indicated by the enhancement factor $f_{\mathrm{H} 98(y, 2)}$ over ELM-free $H$ mode as given by the ITERH98-P $(y, 2)$ scaling law ${ }^{27}$ and by the value of the normalized beta, $\beta_{N}$. In the first discharge (No. 84644) a higher density ramp is pre-programmed, leading a degradation of confinement at the highest fueling rates down to $f_{\mathrm{H} 98(y, 2)}=0.85$. In the second discharge, a lower density

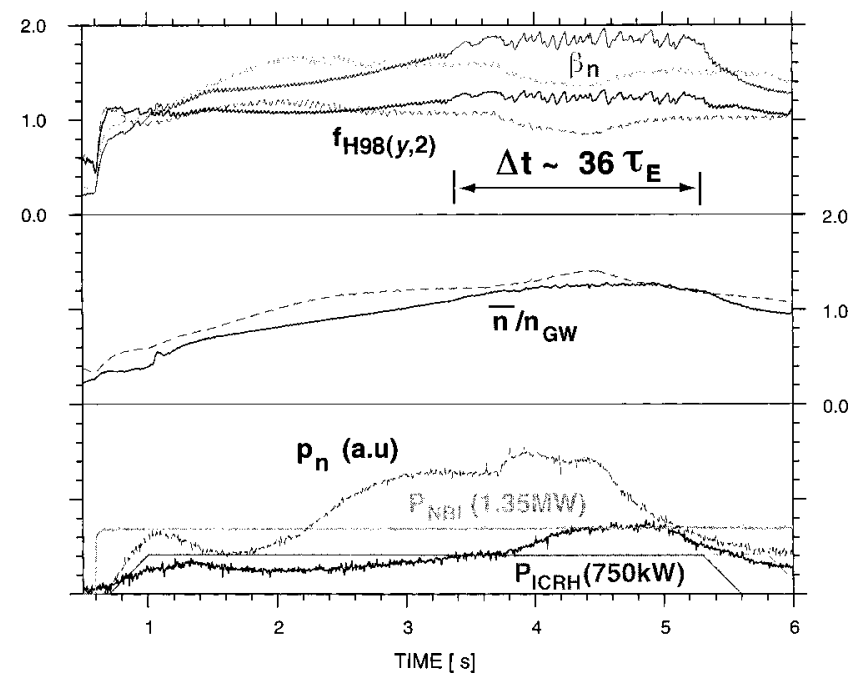

FIG. 2. Illustration of the effect of $\mathrm{D}_{2}$ gas puffing on impurity seeded discharges in TEXTOR-94. In a first discharge moderate gas puffing has been applied (full lines), the second one was fueled by a strong gas puff (dashed lines). Shown as a function of time are the resulting values for $\beta_{N}$, $f_{\mathrm{H} 98(y, 2)}$ and $\bar{n} / n_{\mathrm{GW}} \cdot P_{n}$ is measured in the exhaust duct of ALT-II and is proportional to the edge neutral pressure. Best performance is reached by the discharge with the smallest gas puff and consequently smallest edge neutral pressure. Values for the normalized beta, $\beta_{N} \sim 1.8$, are reached for about $2 \mathrm{~s}$ or $36 \tau_{E}$.

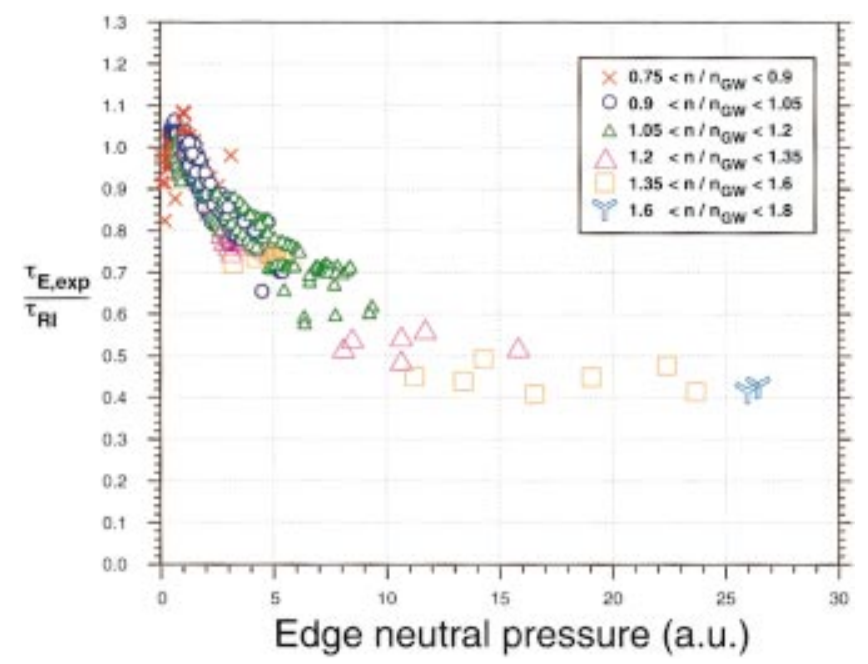

FIG. 3. (Color) Confinement relative to the RI-mode $\tau_{E} / \tau_{\mathrm{RI}}$ vs the edge neutral pressure. Different symbols correspond to the different Greenwald factors reached in the discharges. Surprisingly, a unique relation is found for discharges belonging to a wide range of operational conditions and densities $\left(0.75<\bar{n} / n_{\mathrm{GW}}<1.8\right)$.

ramp and consequently reduced gas puffing rate is applied (No. 84642). Note that, remarkably, even for similar densities (e.g., $t=3 \mathrm{~s}$ in No. 84644 and $t=4 \mathrm{~s}$ in No. 84642) better confinement is nevertheless reached in the discharge with moderate gas puffing, resulting in $f_{\mathrm{H} 98(y, 2)}$ values close to 1.3 at densities $\bar{n} / n_{\mathrm{GW}}=1.1$, whereas the discharge with heavier gas puff never exceeds $f_{\mathrm{H} 98(y, 2)} \sim 1.2$, even at the higher densities reached (up to $\bar{n} / n_{\mathrm{GW}}=1.2$ ). The confinement performance with respect to the RI-mode conditions is strongly linked to the neutral pressure in the plasma edge, as shown in Fig. 3, where the enhancement factor versus RI-mode confinement $\tau_{E} / \tau_{\mathrm{RI}}$ as a function of the edge neutral pressure (in this case measured in the exhaust duct of the pumping limiter ALT-II) is plotted. Remarkably, a unique relationship shows up between those quantities, for discharges in a wide range of densities and performance. The control of the edge neutral pressure is therefore a conditio sine qua non to maintain good confinement at trans-Greenwald densities. In this respect, a feedback system is now planned for TEXTOR-94, adjusting the puffing level in order to maintain the plasma energy constant during density excursions and this should allow for even longer stationary phases at trans-Greenwald densities with high confinement.

\section{RADIATING MANTLE EXPERIMENTS IN DIII-D}

Seeding of impurities has been applied in both ELMy $H$-mode and $L$-mode divertor discharges resulting in improvement of confinement and/or density performance. Of the various configurations considered, ${ }^{28,29}$ we will focus in what follows (i) on lower single null "puff and pump" ELMy $\mathrm{H}$-mode discharges (where a large deuterium gas flow is applied together with the strike points being positioned near the entrance of the divertor cryopumps for particle removal $^{29}$ ) and (ii) $L$-mode upper single null divertor plasmas with $q(0)>1$, and avoid sawtooth which can easily lead to an $L \rightarrow H$ transition. It has been verified that negative 


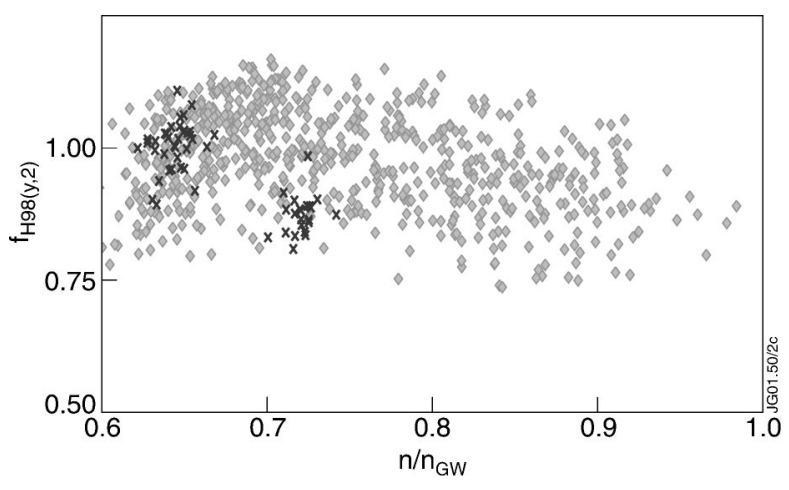

FIG. 4. Overview of confinement obtained in high density ELMy $H$-mode plasmas with puff and pump in DIII-D. Diamonds (gray) represent $\mathrm{Ar}$ seeded discharges, crosses (crosses) unseeded discharges. The presence of the impurity extends the density range for which high confinement is maintained from $n / n_{\mathrm{GW}} \sim 0.75$ up to $n / n_{\mathrm{GW}} \sim 1$.

shear does not contribute to the confinement changes observed. All discharges have been obtained with NBI-co as only additional heating source.

By seeding of Ar in puff and pump ELMy $H$-mode discharges radiating mantles could be established, ${ }^{29}$ and compared to discharges without seeding. The edge pedestal pressure is decreased by about $33 \%$ when increasing the radiating power fraction from 0.4 to $0.8,{ }^{29}$ for a nearly constant enhancement factor $f_{\mathrm{H} 98(y, 2)}$. Impurity seeded puff and pump ELMy $H$-mode discharges allow operation at higher densities compared to unseeded reference discharges, without degradation of confinement, as illustrated in Fig. 4. A reduction has been observed in the effective thermal and toroidal momentum diffusivity (as calculated by TRANSP ${ }^{30}$ ), with a concomitant increase in the thermal neutron production. An increase in toroidal momentum and in the $E \times B$ shearing rate has been seen, contributing to the stabilization of microturbulence, and may be part of the explanation for the decrease in effective thermal diffusivity.

Although not at high density, the results of the $L$-mode divertor plasma with negative shear clearly show the physical mechanisms involved in the stabilization of microinstabilities owing to the impurity seeding. $\mathrm{Ne}, \mathrm{Ar}$, and $\mathrm{Kr}$ seeding have been applied in such plasmas. The observed confinement improvement depends on the quantity of impurities injected and on the impurity used, with the largest effects being observed with Ne. Figure 5(a) illustrates the effect on several plasma parameters for a high and an intermediate level of Ne seeding. A near doubling of the confinement enhancement factor, neutron production, and central ion temperature could be realized. The seeding of impurities results in a modest peaking of the density profile and a reduction in the $D_{\alpha}$ radiation, consistent with an increase in the particle confinement time, as also observed in TEXTOR-94.

Experimentally, confinement enhancement in these $L$-mode divertor discharges is accompanied by a strong reduction in the long wavelength density fluctuations, as measured by beam emission spectroscopy (BES) and far infrared scattering. In addition, a reduced particle flux at the edge as measured with Langmuir probes has been observed. There is
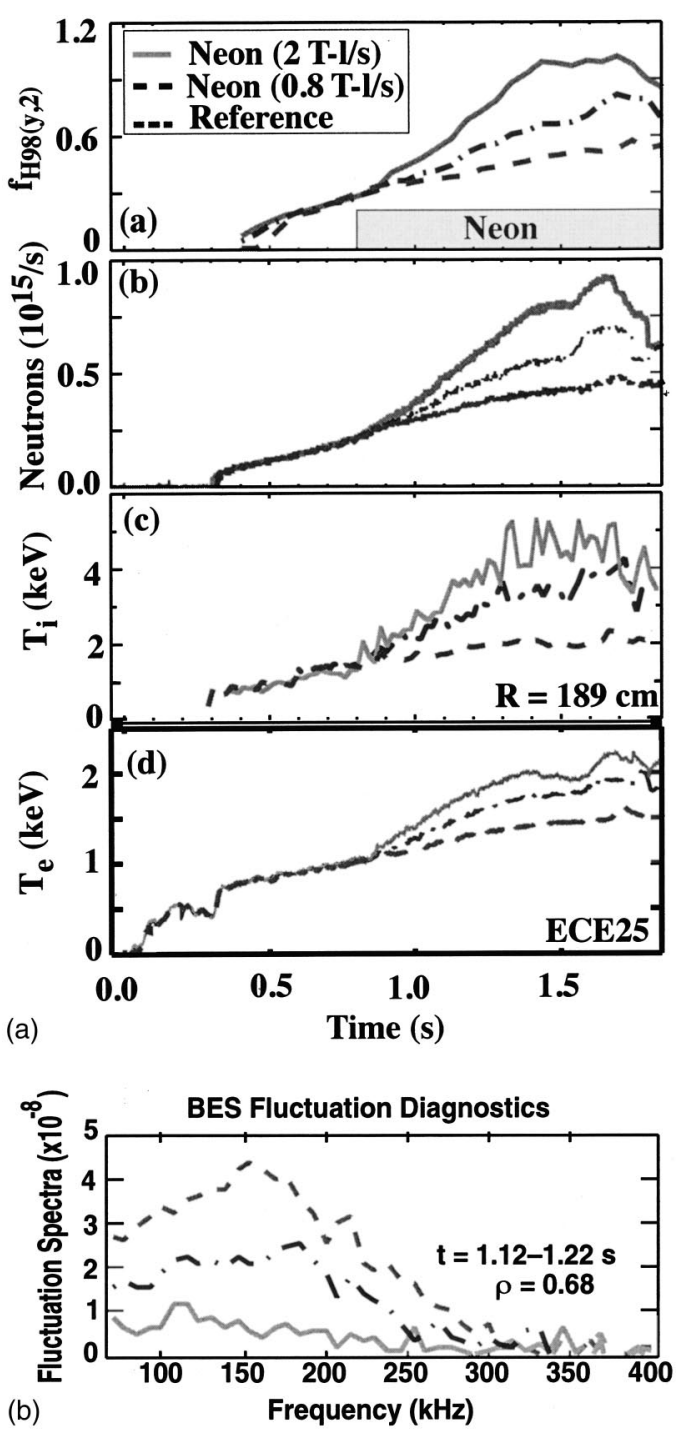

FIG. 5. (a) Illustration of the result of Ne seeding in $L$-mode divertor discharges in DIII-D. Shown are the results obtained for a reference discharge (dashed lines), a discharge with an intermediate (dot-dashed lines), and high level (full lines) of $\mathrm{Ne}$ seeding. For the highest level of seeding a near doubling is seen in the confinement factor $f_{\mathrm{H} 98(y, 2)}$, neutron yield, central ion, and electron temperatures $T_{I}(0)$ and $T_{e}(0)$. Note that the location of the measurement for $T_{i}(0)$ of $R=189 \mathrm{~cm}$ corresponds approximately to $\rho=0.25$ and the one of ECE channel 25 for $T_{e}(0)$ to approximately $\rho=0.36$ (adapted from Ref. 12). (b) Fluctuation spectra as measured by beam emission spectroscopy (BES) for the discharges shown in (a) for $t$ $=1.12-1.22 \mathrm{~s}$ at a normalized radius $\rho=0.68$ (adapted from Ref. 12).

some peaking of the electron density profile observed with impurity injection (as observed in TEXTOR-94). However, gyro-kinetic stability analysis shows that the effect of this peaking on the reduction of the maximum growth rate of the plasma turbulence is modest. During Ne seeding, the toroidal rotation profile changes rapidly, the rotation decreases for $\rho>0.85$ and increases for $\rho<0.85 .^{12}$ This results in an increasing rotation gradient enhancing $E \times B$ shear. This last mechanism appears to be the dominant one for the observed turbulence reduction observed at the beginning of neon seeding. Modeling shows that the physical mechanisms responsible for confinement improvement are impurity induced growth rate reduction of toroidal drift wave turbulence and 
increased radial electric field shear. From TRANSP ${ }^{30}$ analysis, one deduces a reduction of the transport coefficients in all transport channels during impurity seeding: (i) the ion thermal diffusivity at peak performance is reduced to its neoclassical values, (ii) the electron thermal diffusivity reduces modestly (roughly by a factor of 1.5 during Ne seeding), (iii) toroidal momentum diffusivity and particle diffusivity are also decreased. Central $Z_{\text {eff }}$ values increase (e.g., for $\mathrm{Ne}$ seeding from 1.5 to 3.4). However, the central deuteron density remains nearly the same, because of the simultaneous decrease in the intrinsic carbon density and the peaking of the density. The observed higher neutron yield is consistent with the increase in the ion temperature.

In order to create conditions as close as possible to those of TEXTOR-94 and to investigate the possible influence of the plasma elongation on the confinement changes with impurity seeding, inner wall limited discharges with a low elongation $(\kappa \sim 1.2)$ [see Fig. 6(a)] have been developed on DIII-D. ${ }^{29}$ Impurity seeding in these plasmas led to a behavior that is qualitatively similar to the TEXTOR-94 RI-mode discharges, with marked improvements in energy and particle confinement as illustrated in Fig. 6(b). BES measurements show also in these discharges a marked reduction in the long wavelength density fluctuations [Fig. 6(c)], and thus indicate that the same mechanisms are at work as in the $L$-mode divertor discharges.

\section{RADIATING MANTLE EXPERIMENTS IN JET}

Radiative mantle plasmas have been realized on JET in $L$-mode limiter plasmas, ${ }^{15}$ in $L$-mode divertor plasmas, ${ }^{16}$ and in ELMy $H$-mode plasmas. ${ }^{14,17}$ In what follows we will restrict the discussion to the results obtained in the latter plasmas, because it allows extending good confinement at higher densities, close to the Greenwald density limit. The $X$ point has been lowered in those discharges onto or just inside the material barrier (called septum or dome) dividing the inboard and outboard sides of the divertor, resulting in the so-called "septum" configuration. (see Fig. 7). This configuration thus represents a combination of a divertor and pumped limiter configuration. Due to the lower $H$-mode power threshold, ${ }^{31}$ these plasmas have the added advantage to stay in $H$ mode even at high radiation levels. Figure 8 illustrates a typical time sequence for such a discharge. After the start of the additional heating, a strong gas puff is applied in order to increase the density. Confinement is strongly reduced in this phase due to the strong gas puffing as also observed on TEXTOR-94 and in other plasma operational scenarios, e.g., the ones used in D-T experiments in TFTR. ${ }^{32}$ The confinement degradation is clearly shown in the reduction of the enhancement factor $f_{\mathrm{H} 98(y, 2)}$, decreasing from 1.13 to 0.76 . During the $\mathrm{D}_{2}$ puffing phase, additionally Ar seeding leads to an increase in the radiative power level and in the plasma density, as a result of an increase in the particle confinement time during impurity seeding, as observed on TEXTOR-94. After the closure of the $\mathrm{D}_{2}$ gas fueling valve, the density remains the same, but confinement is restored, and in this way we reach outstanding values for the product $f_{\mathrm{H} 98(y, 2)} \bar{n} / n_{\mathrm{GW}} \sim 1.0$ at $\bar{n} / n_{\mathrm{GW}} \sim 0.9$ and radiation fractions (a)
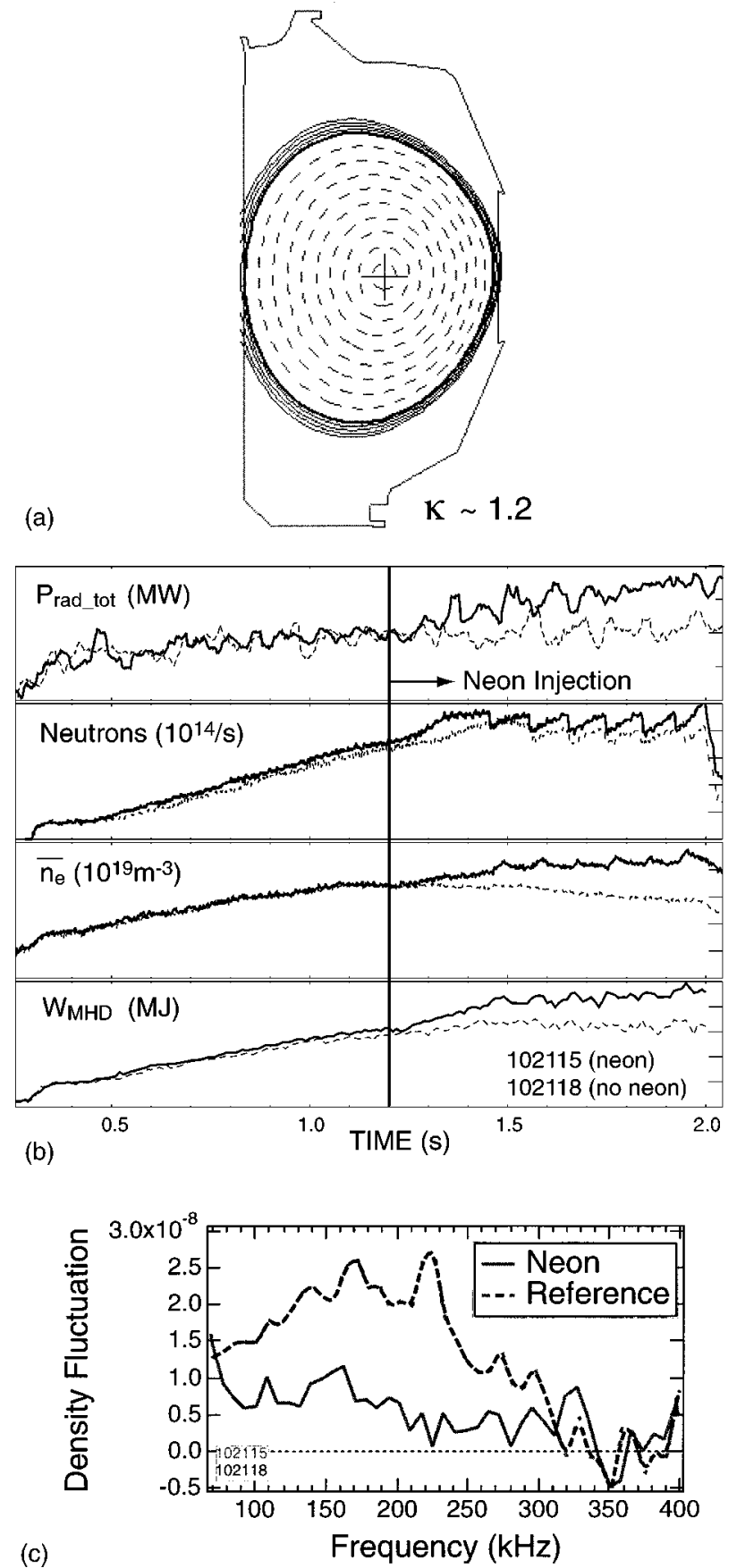

FIG. 6. (a) Plasma shape for near circular discharges in DIII-D with an elongation $\kappa \sim 1.2$. (b) Influence of $\mathrm{Ne}$ seeding on the radiation, neutron yield, density and stored plasma energy in nearly circular discharges in DIII-D. Full lines correspond to the discharge with $\mathrm{Ne}$ seeding (No. 102115), dashed lines to the reference discharge without Ne seeding (No. 102118). (c) Fluctuation spectra as measured by beam emission spectroscopy for nearly circular discharges of (b).

$P_{\text {rad }} / P_{\text {tot }}$ up to $60 \%$. Those values can last for three to four confinement times. This phase is called in what follows the "afterpuff" phase. During this afterpuff phase, careful Ar and $\mathrm{D}_{2}$ puffing is applied to keep the radiation level and the plasma density as constant as possible. For the discharges under consideration most of the radiation is localized in the edge and not in the divertor and/or $X$ point, typically $P_{\text {divertor }} / P_{\text {tot }} \sim 15 \%-20 \%$ for impurity seeded ELMy 


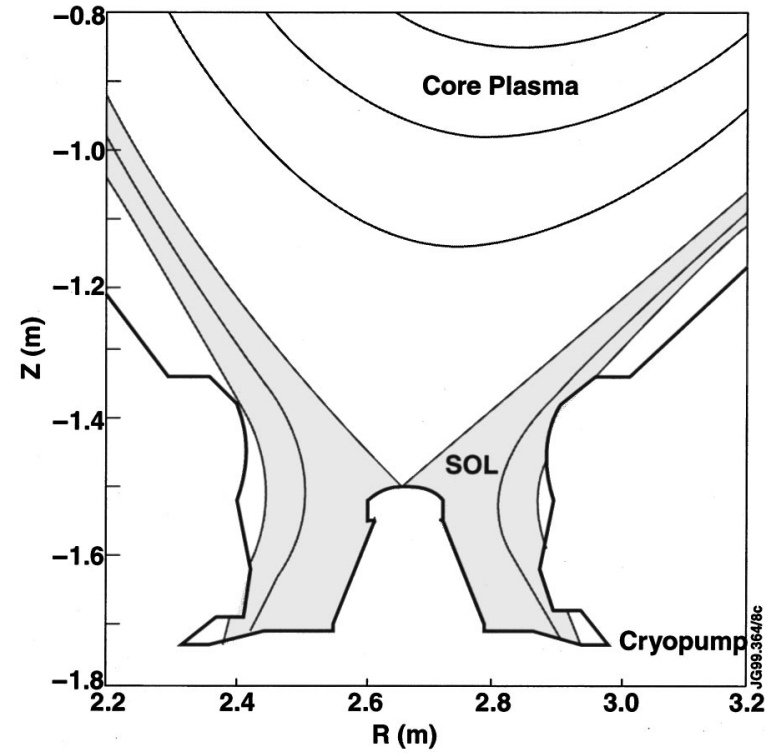

FIG. 7. JET divertor with barrier ("septum" or "dome") separating the inner from the outer divertor leg. In so-called "septum" discharges, the $X$ point is located on top of or slightly inside the septum.

$H$-mode shots in the septum configuration. Ar seeding is more adapted to the JET plasma parameters than $\mathrm{Ne}$, as the latter leads for a given radiation level to a higher $Z_{\text {eff }}$.

The presence of the impurity not only increases radiation, but can also change the character of the ELMs. Indeed

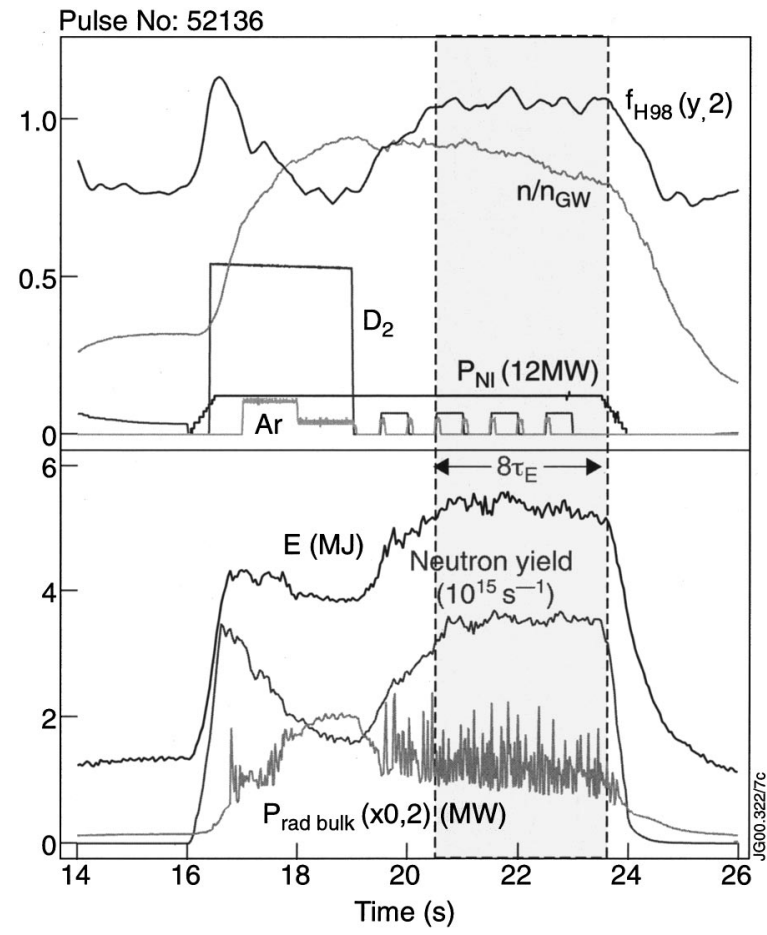

FIG. 8. Time traces for a typical impurity seeded discharge in JET, with a so-called "afterpuff" phase. Shown as a function of time are the confinement factor $f_{\mathrm{H} 98(y, 2)}$, the Greenwald factor, neutron yield, bulk radiation, and deuterium and Ar puffing. The main puffing phase is followed by the so-called "afterpuff" phase (consisting in this case in a mixture of carefully chosen levels for $\mathrm{D}_{2}$ and Ar puffing), during which high confinement and high density is simultaneously realized. a difference has been observed in the effect of the impurity on the character of the ELMs if applied during the $\mathrm{D}_{2}$ puffing phase or during the afterpuff. Impurity seeding (by $\mathrm{Ar}$ or $\mathrm{Ne}$ ) applied during the main $\mathrm{D}_{2}$ puffing phase can change the ELMs from type I to more benign high frequency type III ELMs. Surprisingly, impurity seeding applied in the afterpuff gives rise to opposite effects reducing the frequency of type I ELMs, and can, with sufficient Ar seeding, lead to intermittent ELM-free phases, and a tendency to impurity accumulation. Impurity seeding thus provides an additional knob to change the behavior of the ELMs, and thus possibly is a means to optimize in a general way the properties of the $H$ mode.

The fact that impurity seeding during the puff is able to further increase the plasma density is illustrated in Fig. 9(a) for two discharges with and without Ar during the $\mathrm{D}_{2}$ puff, but otherwise the same operational plasma parameters. The discharge with Ar puffing shows during the $\mathrm{D}_{2}$ puff a $10 \%-$ $15 \%$ higher density compared to the shot without impurity puffing, and reaches $\bar{n} / n_{\mathrm{GW}} \sim 95 \%$. Due to the higher density reached, $Z_{\text {eff }}$ is not increased but is even somewhat lower in the discharge with impurity puffing, and reaches a value down to about 2. As confinement is nearly the same in both cases, this example illustrates clearly that impurity seeding allows one to increase the product $f_{\mathrm{H} 98(y, 2)} \bar{n} / n_{\mathrm{GW}}$ up to 1.0 . Part of the confinement changes during Ar seeding at a given (high) density are due to the change in the character of the ELMs as discussed previously: reduction of the ELM frequency, due to the presence of Ar, with a similar pedestal, resulting in smaller losses via ELMs. Nevertheless, fundamental changes in bulk confinement must also take place, as indicated by a clear drop in $\chi_{\text {eff }}$ (calculated by TRANSP ${ }^{30}$ ) in shots with Ar seeding, illustrated in Fig. 9(b). Figure 10 gives an overview of the data obtained in ELMy $H$-mode septum afterpuff discharges and compares them with previous JET data obtained without impurity seeding in the various divertor configurations tested up to the end of 1999. It is clear that the data points with impurity seeding are exceeding those without impurity seeding, with outstanding values for the product $f_{\mathrm{H} 98(y, 2)} \bar{n} / n_{\mathrm{GW}} \sim 1.0$ at densities around $\bar{n} / n_{\mathrm{GW}}$ $=0.9$. Such phases have been obtained stationarily in JET for durations of about $1.2 \mathrm{~s}$ or $3 \tau_{E}$. Quasistationary phases with a slow reduction in density by about $20 \%$ but otherwise constant values for confinement and radiation, have been obtained for about $8 \tau_{E}$. Further experiments are aimed at realizing longer stationary phases for the density, radiation level, and confinement.

\section{RADIATING MANTLE EXPERIMENTS IN JT-60U}

In JT-60U $\mathrm{Ar}^{11}$ seeding has been applied in ELMy $H$-mode discharges. Note that this machine is equipped with a W-shaped divertor, composed of a dome, inner and outer divertor plates. Recently, feedback controlled Ar seeding has been applied in the main chamber in high triangularity $(\delta$ $>0.35$ ) ELMy $H$-mode plasmas, heated by neutral beam $(16-20 \mathrm{MW})$ at a plasma current $I_{p}=1.2 \mathrm{MA}$, toroidal magnetic field $B_{t}=2.5 \mathrm{~T}$, and $q_{\text {edge }} \sim 3.4$. In this way conditions could be realized with simultaneously a high radiation frac- 
Pulse No: 50344,50337
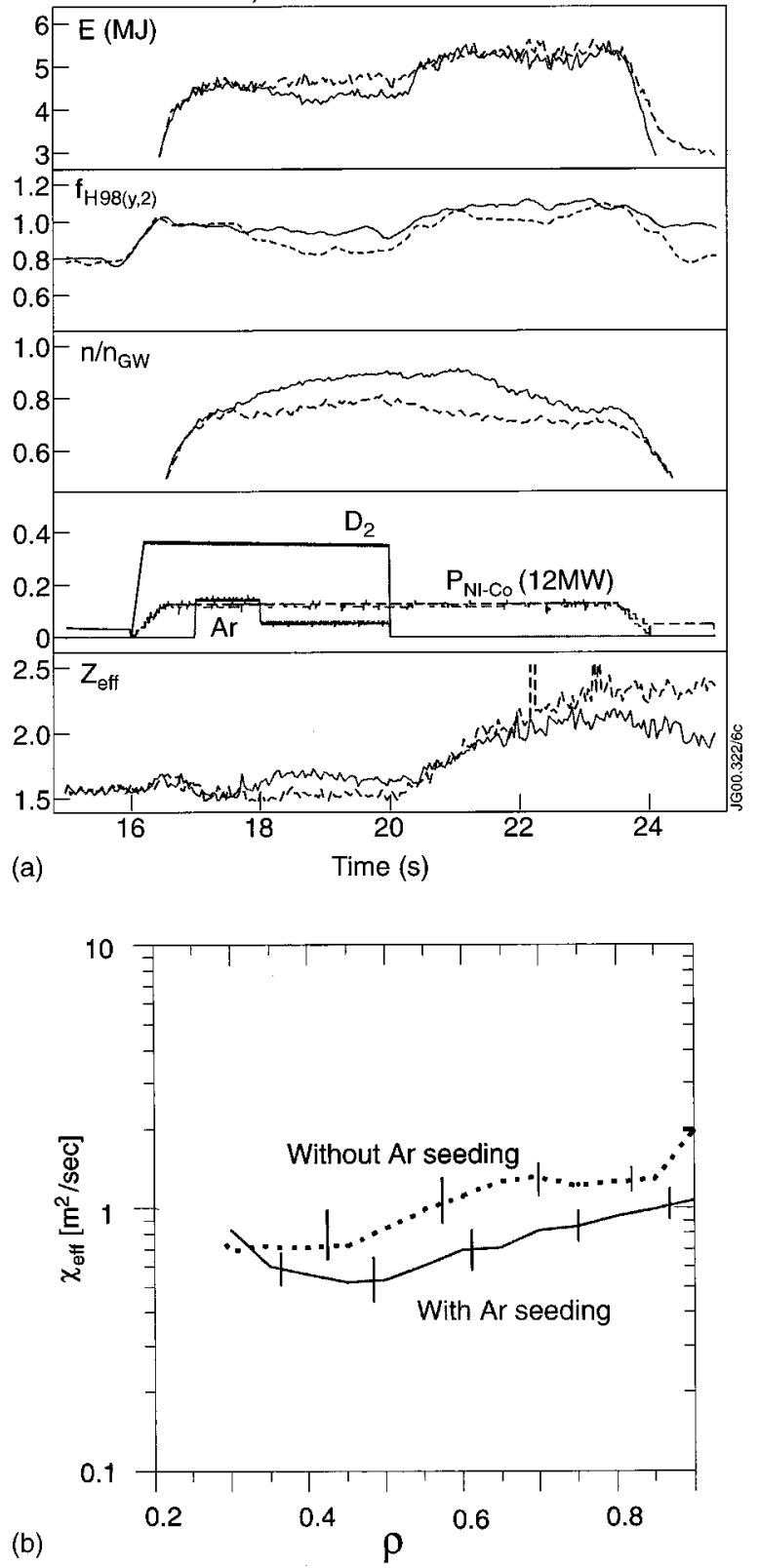

FIG. 9. (a) Comparison of two "afterpuff" discharges in JET with (full lines) and without (dashed lines) Ar impurity seeding. Shown as a function of time are the plasma stored energy $E$, confinement factor $f_{\mathrm{H} 98(y, 2)}$, Greenwald factor, effective plasma charge $Z_{\text {eff }}$, and gas puffing scheme. Note that the discharge with impurity seeding reaches a lower $Z_{\text {eff }} \sim 2$ in the "afterpuff" phase, showing clearly the overwhelming effect of the density increase on the plasma dilution. Note that the zeros have been suppressed in all but one of the ordinates. (b) Comparison of the radial profile of the effective thermal diffusivity $\chi_{\text {eff }}$ during the "afterpuff" ( $\left.t=21 \mathrm{~s}\right)$ for the two discharges of (a). The dashed curve refers to the discharge without, the full curve to the discharge with Ar seeding. Error bars are indicated by the small vertical bars. The discharge with Ar seeding shows a net reduction in $\chi_{\text {eff }}$ over most of the plasma radius.

tion $P_{\text {rad }} / P_{\text {tot }} \sim 80 \%$, densities $\bar{n} / n_{\mathrm{GW}} \sim 0.65$, and high confinement $f_{\mathrm{H} 98(y, 2)}=1$ for a duration of about $2.5 \mathrm{~s}$. Furthermore, Ar seeding leads to an increase in stored energy of about $40 \%$ at a density of $\bar{n} / n_{\mathrm{GW}} \sim 65 \%$, and higher neutron yields are observed. The radiation profile shows the formation of an edge radiating layer. An $X$-point MARFE is usu-

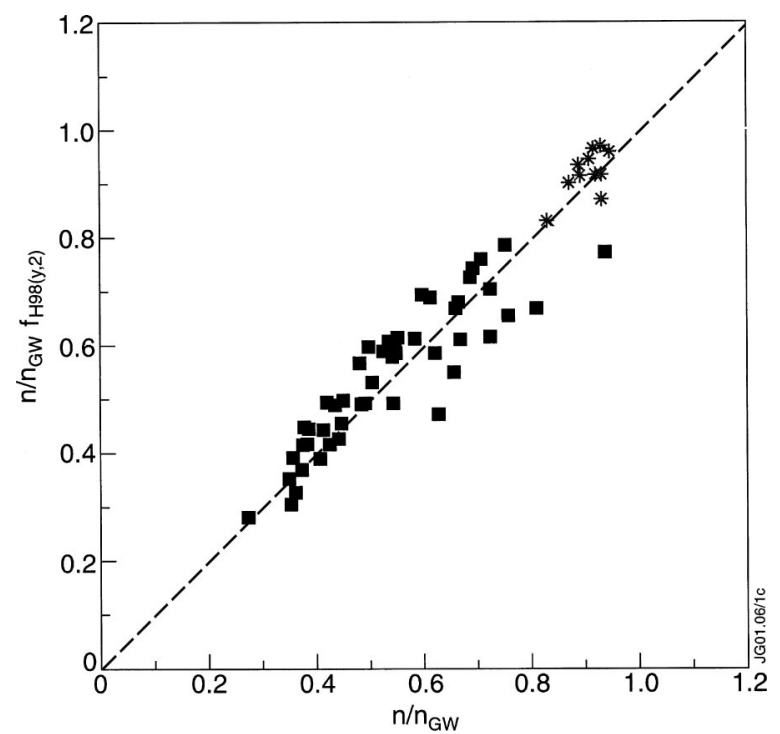

FIG. 10. Plot of the product $f_{\mathrm{H} 98(Y, 2)} \bar{n} / n_{\mathrm{GW}}$ vs $\bar{n} / n_{\mathrm{GW}}$ for JET. Squares indicate JET discharges without impurity seeding and are selected from the dataset contributed to the ITER confinement database (including data from discharges up to the end of 1999). Stars indicate discharges with impurity seeding from recent campaigns. Outstanding values of $f_{\mathrm{H} 98(y, 2)} \bar{n} / n_{\mathrm{GW}} \sim 1.0$ at $\bar{n} / n_{\mathrm{GW}} \sim 0.9$ have been reached recently in impurity seeded discharges. All data are selected according to $0.175<\delta<0.3,2.3<\operatorname{Ip}(\mathrm{MA})<2.6,2.3$ $<\operatorname{Bt}(\mathrm{T})<2.6,9<\operatorname{Pin}(\mathrm{MW})<14$.

ally seen at densities above $\bar{n} / n_{\mathrm{GW}}=50 \%$, independent of $\mathrm{Ar}$ seeding, and is the dominant source of radiation in the absence of Ar. With Ar seeding, however, up to $60 \%$ of the radiation losses are located in the main chamber. Typical plasma profiles are shown in Fig. 11. For a given density profile, higher central temperature profiles are found with steeper gradients, indicative of fundamental confinement changes in the core. Also the plasma edge is affected by the presence of the impurity as a higher ion temperature is measured at the pedestal. In fact, there is a relation between the edge temperature and the confinement improvement and this
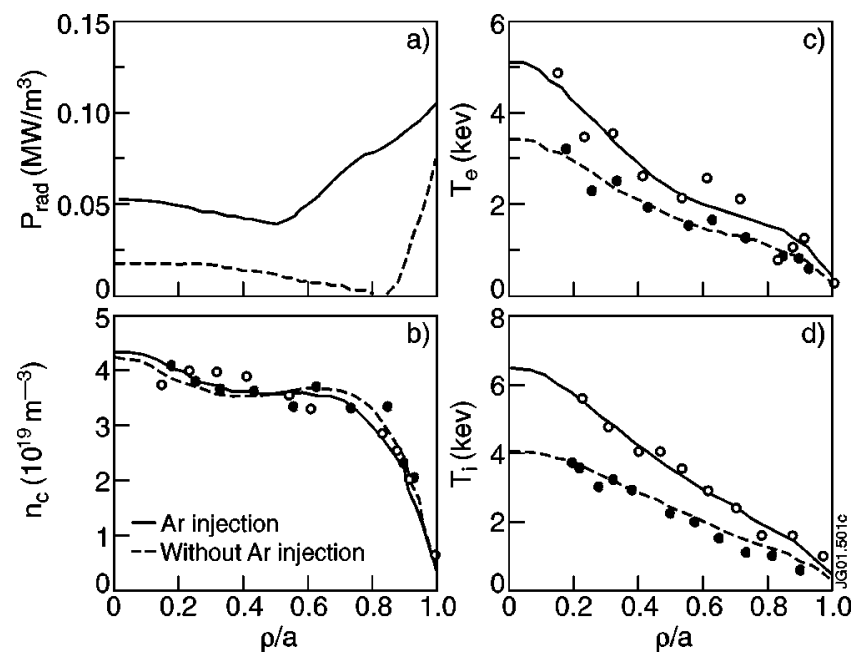

FIG. 11. Effect of impurity seeding on plasma profiles in JT-60U. Shown are the profiles of the radiated power, plasma density, and ion and electron temperature. A clear increase in the central temperatures is seen, indicating a genuine core confinement improvement (adapted from Ref. 13). 


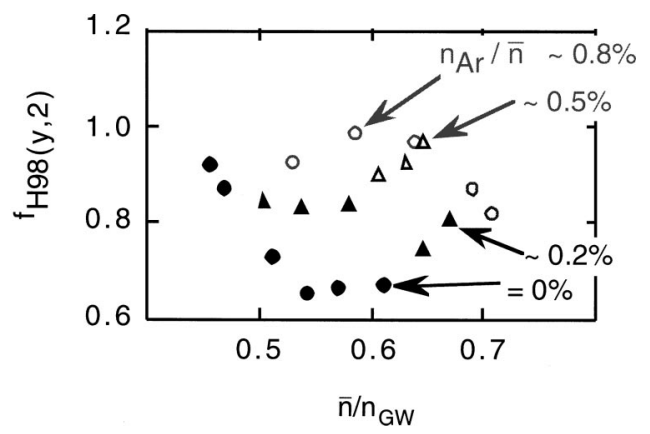

FIG. 12. Confinement factor $f_{\mathrm{H} 98(y, 2)}$ vs $\bar{n} / n_{\mathrm{GW}}$ for discharges in JT60-U with different amounts of Ar seeding. Black dots refer to discharges without $\mathrm{Ar}$ seeding. The highest levels of Ar seeding lead to the realization of $f_{\mathrm{H} 98(y, 2)}=1$ up to $\bar{n} / n_{\mathrm{GW}}=0.65$ (adapted from Ref. 13).

suggests temperature profile stiffness attributed to the ITG mode. As on JET with Ar seeding in the absence of a $D_{2}$ puff, there is an obvious reduction of the frequency of the ELMs, as soon as $\mathrm{Ar}$ is introduced in the discharge. Both these edge and core effects clearly show again the fundamental changes induced in plasma confinement by the presence of the radiating impurity. These changes are thought to be related to the stabilization of ITG modes, and further work is underway to elucidate the precise mechanism.

An overview of the confinement obtained is given in Fig. 12. Different symbols reflect different levels of Ar seeding. At the highest levels $\left(n_{\mathrm{Ar}} / \bar{n} \sim 0.5 \%\right)$ ELMy $H$-mode confinement $\left(f_{\mathrm{H} 98(y, 2)}=1\right)$ can be extended to $\bar{n} / n_{\mathrm{GW}}=65$. At densities above $\bar{n} / n_{\mathrm{GW}}=75 \%$, the plasma is very sensitive to even tiny amounts of impurities, and radiative collapses easily occur. Up to now this prevented a further extension of improved confinement to even higher densities. One way to counteract such collapses would be to increase the central electron temperature. To try this a more elaborate feedback control for impurity seeding, involving a feedback on the central heating, is now under development.

\section{THEORETICAL UNDERSTANDING OF CONFINEMENT PHYSICS IN RADIATING MANTLE PLASMAS}

\section{A. Simulation of turbulence with three-dimensional gyrokinetic codes}

The physical mechanisms of turbulence suppression and anomalous transport reduction in RI-mode plasmas have been investigated with nonlinear gyrokinetic particle simulations in toroidal geometry, including global profile variation effects and multiple ion species. ${ }^{33,34}$ Both $L$-mode and RImode TEXTOR-94 plasmas have been simulated in the linear and nonlinear (i.e. saturated) state of the turbulent fluctuations. From the simulations, direct stabilization of ITG turbulence and improved ion thermal confinement has been found in neon-seeded discharges. The highest charge states of Ne induce a stabilizing effect on the linear growth of ITG modes by factors of 2 to 3 . In addition, during the RI-mode phase radiation cooling results in a further reduction of the turbulence since the temperature profiles adjust to become closer to the marginally stable ones for ITG turbulence. This is illustrated in Fig. 13 that shows the spatial distribution of the turbulence and the reduced levels in going from $L$-mode initial profiles to RI-mode profiles taken immediately after the transition. The stabilizing effects of neon were also found to be sensitive to the level of edge turbulence. When the edge ion temperature profile gradient was increased locally the stabilizing effect of the neon impurity on the ITG modes in the linear phase did not persist in the nonlinear phase. In addition, two other important effects were uncovered in the TEXTOR case. The first is the $E \times B$ shearing rate from the combined effects of the equilibrium radial electric field and the turbulence-induced fluctuating radial electric field. When the growth rate of the ITG modes is reduced to levels comparable to the $E \times B$ shearing rate, the suppression effects were much stronger. Second, there is also sensitivity to the impurity concentration. With increased impurity seeding, (i) stronger stabilizing effects are observed on ITG turbulence levels and (ii) larger profile adjustments from radiation cooling.

In order to understand whether these results could extend to plasmas which have a weaker collisionality and smaller $\rho^{*}=\rho_{i} / a$ (with $\rho_{I}$ the ion Larmor radius) we have made a numerical experiment consisting in using profiles from TEXTOR-like $L$-mode and RI-mode discharges but with doubled electron and ion temperatures. The weaker collisionality with higher temperatures reduces the linear growth rate of the ITG modes by about $10 \%$. Without the addition of impurities the saturated fluctuation amplitude of the turbulence is approximately the same in both the regular TEXTOR-94 case and the one with doubled temperatures. There are two reasons for this. First, the collisionality dependence of the ion thermal transport is found to be weak in the $L$-mode regime. ${ }^{35}$ Second, ion thermal transport is found to be Bohm-like in the $L$-mode regime, exhibiting a weak dependence on $\rho^{*}$ and this is found both experimentally (using dimensional similar discharges) and in simulations. ${ }^{33,35}$ When Ne is introduced into the TEXTOR-94 plasmas with a doubled temperature, a reduction in the saturated fluctuation level and ion thermal transport is observed, similar to the regular TEXTOR-94 results. We conclude that a lower value for the collisionality (as it is for larger machines or in a reactor), cannot inhibit the stabilizing effect of the seeded impurity.

\section{B. Reduction of ITG turbulence}

In the fluid approximation, the surprising effect of impurity seeding as seen in TEXTOR-94 RI-mode plasmas can also be understood as caused by the reduction of ITG turbulence. According to the model in Ref. 24, a simplified expression for the linear ITG growth rate is given by

$$
\gamma_{\mathrm{ITG}} \propto \sqrt{\frac{\epsilon_{i}}{Z_{\mathrm{eff}}}\left(1-\frac{0.67}{\eta_{i}}\right)-\frac{\epsilon_{i}}{4 \eta_{i}^{2}}}
$$

with $\epsilon_{I}=-R / 2(d \ln T i / d r)$ characterizes the gradient of the ion temperature, $\eta_{i}=d \ln n_{i} / d \ln T_{i}$ determines the peaking of the ion temperature profile, $R$ and $r$ are the major and minor radius of the plasma. An increase in $Z_{\text {eff }}$, brought about by the impurities, reduces the ITG turbulence in this model. 


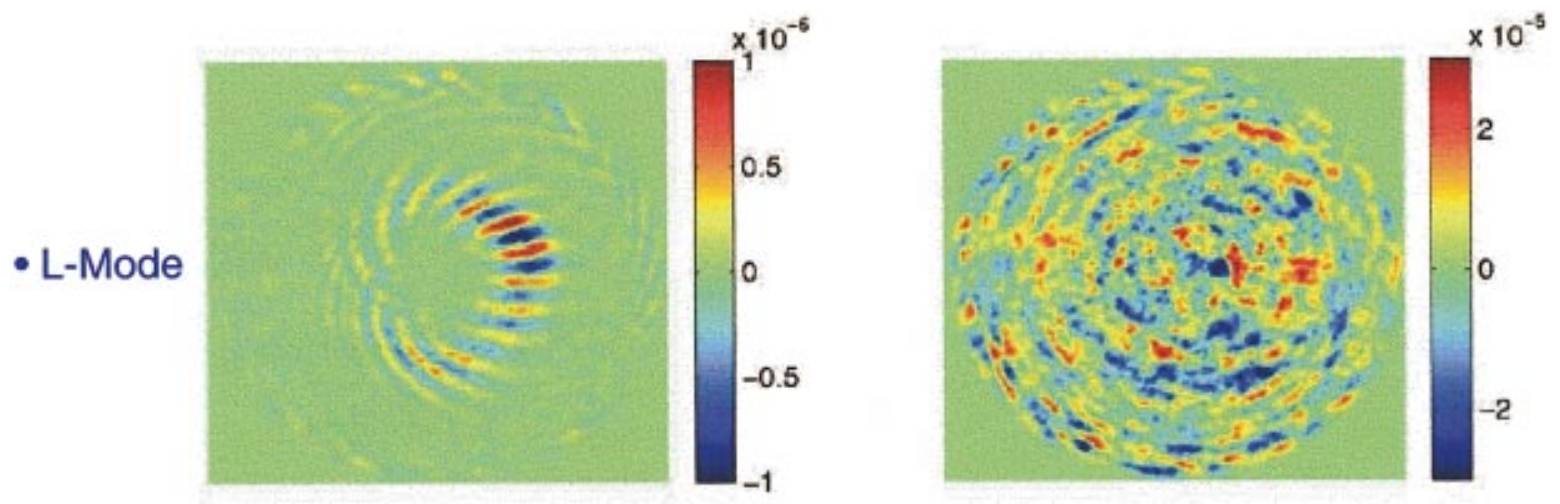

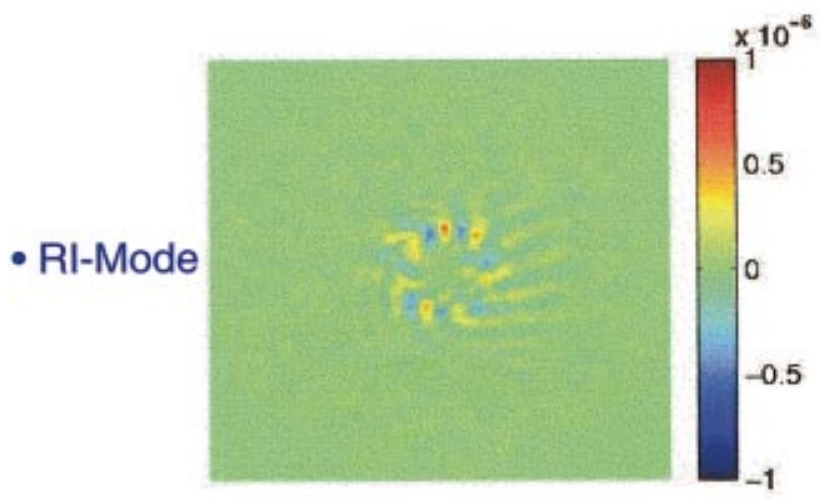

- Linear phase

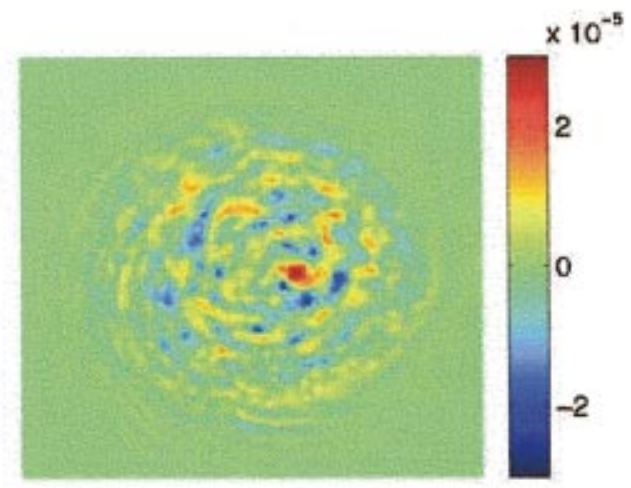

- Saturated phase

FIG. 13. (Color) Electrostatic potential fluctuations as found in 3-D nonlinear gyrokinetic calculations using measured plasma profiles. Shown are the results obtained for a L-mode and RI-mode shot, in the linear and saturated turbulence phase. Note the larger scale used to plot the saturated phase, showing the importance of saturated turbulence over the linear one. The reduced level of turbulence during the impurity seeding in the TEXTOR-94 RI mode is clearly seen.

Once the damping of the ITG modes sets in, a nonlinear amplification of the quenching of the ITG modes occurs, due to the dependence on $\eta_{i}$. Indeed, the reduction of the ITG modes increases the relative importance of the dissipative trapped electron (DTE) mode turbulence in the plasma, with a growth rate $\gamma_{\mathrm{DTE}}$ proportional to $1 / \eta_{I}$.

To describe the evolution of the profile peaking in TEXTOR RI-mode discharges, we introduce the function

$$
\mathrm{G}\left(\eta_{I}\right)=\Gamma\left(\eta_{i}\right)-\frac{1}{r} \int_{0}^{r} S_{e}\left(r^{\prime}\right) r^{\prime} d r^{\prime}
$$

For a given plasma minor radius, this function must be equal to zero in steady state to satisfy the continuity equation for the electrons at that radius. In this model, the dominating transport channels are considered to be due to ITG and DTE modes and then $\Gamma=\Gamma_{\mathrm{DTE}}+\Gamma_{\mathrm{ITG}}$. The function $\mathrm{G}\left(\eta_{I}\right)$ is shown for a particular TEXTOR RI-mode discharge at a given radius in Fig. 14. At low $Z_{\text {eff }}$, the function $\mathrm{G}\left(\eta_{I}\right)$ has three solutions, of which only the one corresponding the highest $\eta_{I}$ describes the $L$-mode state. There are two ways in which this function can produce only one solution with low $\eta_{I}$ values. First, for a given source term, the maximum value of $\mathrm{G}\left(\eta_{I}\right)$ decreases with increasing $Z_{\text {eff }}$ and for values exceeding a certain threshold, a spontaneous bifurcation takes place, characterized by a low value for $\eta_{I}$, corresponding to the RI-mode of TEXTOR-94. Second, for a given $Z_{\text {eff }}$, an increase in the source term also reduces the value of $\mathrm{G}\left(\eta_{I}\right)$ and for a sufficiently strong source term, only the solution with the lowest $\eta_{I}$ remains. In practical terms, this means

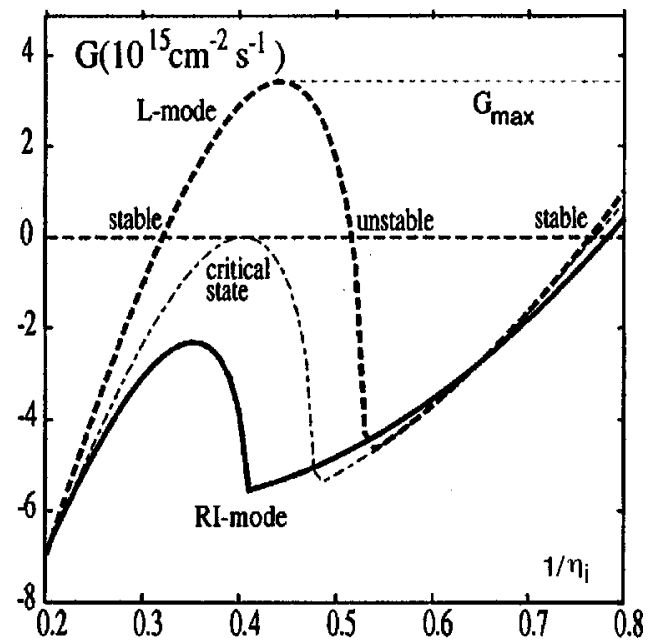

FIG. 14. The function $\mathrm{G}\left(\eta_{I}\right)$ plotted vs $1 / \eta_{I}$ at a minor radius $r=30 \mathrm{~cm}$ of TEXTOR discharge 68803. Depending on the value of the bulk particle source term and $Z_{\text {eff }}$, this function can bifurcate from a solution at a low to a high peaking factor (adapted from Ref. 24). 
that an increase in $Z_{\text {eff }}$ or a sufficiently large source term are needed to realize the peaking required for the RI mode. Note that it is not straightforward to extrapolate the predictions of this theoretical description to reactor-like machines, with a much lower collisionality, as the dependence of $\gamma_{\text {DTE }}$ varies with collisionality. This results in another expression for the function $\mathrm{G}\left(\eta_{I}\right)$, leading to another behavior of the solutions of this equation. This is currently under investigation.

\section{Canonical profiles transport model}

It is in this context interesting to indicate a totally different approach to the two models mentioned previously. The basic idea of the canonical profiles transport model ${ }^{36}$ is the "profile consistency principle" as first proposed by Coppi $^{37}$ and confirmed later in a large number of experiments. Instead of trying to explain the various details of plasma transport, "canonical plasma profiles"' are proposed in this model for the poloidal magnetic field, which in turn determine the profiles for temperature and density. Anomalous values for the transport coefficients appear in the model when the real plasma profiles deviate from the canonical ones. The differential equations for the canonical profiles are found with appropriate boundary conditions. To describe the RI mode a change in the standard boundary conditions is necessary and leads to a change of the canonical profiles such that the edge values of the plasma parameters have a determining influence on the core temperature and density. A detailed account on the description of the RI mode with this model can be found in Ref. 38 .

\section{CONCLUSIONS}

Radiative mantle plasma experiments are being pursued in all large tokamaks around the world. Although the magnitude of the effects observed are not yet at the same level as observed in TEXTOR-94, all experiments described in this paper, either in limiter or divertor configuration, have shown that impurity seeding results in either (i) an increase of confinement at a given density or (ii) enhanced confinement at higher densities. For some machines this resulted in an ELM-free $H$-mode confinement quality close to or even beyond the Greenwald limit. In contrast to strong deuterium puffing which leads to energy and particle confinement rollover, impurity seeding can lead to energy and/or particle confinement improvement. ${ }^{39}$ The primary candidate explanation of the confinement improvement with impurity seeding is stabilization of the ITG modes in the plasma bulk due to the presence of the impurity with the accompanied density peaking and/or - as seen on some machines - an increase in the $E \times B$ shearing rate [for more details see Ref. 40], resulting from a steeper toroidal velocity profile. TEXTOR-94 has also shown the decisive influence of gas fueling on the confinement properties of RI-mode discharges resulting from an increase of fluctuations close the to last closed magnetic surface, and has used this to advantage in order to reach high confinement $\left(f_{\mathrm{H} 98(y, 2)} \geqslant 1.3\right)$ at the highest densities $\left(\bar{n} / n_{\mathrm{GW}}\right.$ $=1.4)$.

In JET, outstanding values for the product $f_{\mathrm{H} 98(y, 2)} \bar{n} /$ $n_{\mathrm{GW}} \sim 1.0$ have been obtained at $\bar{n} / n_{\mathrm{GW}} \sim 0.9$, with Ar seed- ing in ELMy $H$-mode divertor "septum" plasmas, for durations of about $3 \tau_{E}$ in the "afterpuff" phase. The radiation is peaking in the plasma periphery with only a small part localized in the divertor: about $15 \%-20 \%$ of the total radiation, including the $X$ point. Further investigations aim at extending the stationarity of the density during the high performance afterpuff phase.

In JT-60U, radiating mantle plasmas have been obtained by seeding Ar in the divertor, and have also allowed one to extend high confinement conditions of ELMy $H$-mode plasmas $\left(f_{\mathrm{H} 98(y, 2)} \sim 1\right)$ to higher densities $\left(\bar{n} / n_{\mathrm{GW}} \sim 0.65\right)$. As in JET, Ar injection leads to the creation of a radiating mantle, and to an increase in confinement in both the pedestal and the bulk plasma. For a given density, the plasma temperature is increased, showing a fundamental change in bulk confinement.

In DIII-D, impurity seeding combined with specialized techniques for fueling and exhaust it has been possible to obtain stationary ELMy $H$-mode discharges with $f_{\mathrm{H} 98(y, 2)}$ $\sim 1$ at densities near the Greenwald limit. Dedicated studies with extensive diagnostic coverage in $L$-mode divertor plasmas at lower densities have clearly demonstrated a strong reduction of turbulence resulting from impurity seeding. Similar studies are underway in JET $L$-mode divertor plasmas for comparison and first results indicate the occurrence of similar phenomena, although confinement enhancement in these $L$-mode limiter discharges are limited by the early onset of magnetohydrodynamic activity, i.e., ELMs, sawteeth, or $n=2$ modes. In addition, experiments have been dedicated to nearly circular $(\kappa \sim 1.2)$ inner wall limited plasmas in DIII-D to study the influence of the plasma shape and to link with TEXTOR-94 RI-mode plasmas. Also in this case a clear reduction in core turbulence has been observed during impurity seeding.

Two classes of models are used in the theoretical interpretation of radiative mantle discharges. A first class, consisting in a detailed description or numerical simulation of turbulent plasma processes, and a second class based on a minimal energy principle. The first show a reduction due to the presence of the impurity of toroidal drift wave turbulence, via the increase in $Z_{\text {eff }}$ and peaking of the density profile and/or increase in the $E \times B$ shear rate. Another important mechanism to reach high density is also the effect of the impurity on the particle confinement time and on the reduction of turbulence associated with the pressure of main gas neutrals at the plasma edge. The second class uses a description based upon the minimization of a free energy functional to determine so-called "canonical profiles," and the deviation of the real plasma profiles from the canonical profiles determines anomalous transport. In this last model, the RI mode can only be described if there is a strong influence from the plasma edge on the final shape of the profiles, in contrast to the $L$ mode where the edge plays a minor role in determining these.

In conclusion, it has been shown in the past years that impurity seeding has resulted in a significant increase in plasma performance in the larger machines DIII-D, JT60-U, and JET. This is a remarkable result, given the variation in size and the differences in the operational details of the dif- 
ferent tokamaks. We are opening here a fully unexplored branch of tokamak physics with potential applications for an integrated operational regime of a future fusion reactor.

\section{ACKNOWLEDGMENTS}

The JET results in this paper have been performed in part under the JET Joint Undertaking and in part under the European Fusion Development Agreement.

${ }^{1}$ M. Greenwald, J. L. Terry, S. M. Wolfe et al., Nucl. Fusion 28, 2199 (1988).

${ }^{2}$ J. Ongena, A. Messiaen, G. Van Wassenhove et al., Controlled Fusion and Plasma Physics, Proceedings of the 20th European Conference, Lisboa, 1993 (European Physical Society, Geneva, 1993), Vol. 17C, Part I, pp. 127-130.

${ }^{3}$ A. M. Messiaen, J. Ongena, U. Samm et al., Phys. Rev. Lett. 77, 2487 (1996).

${ }^{4}$ R. Weynants, A. Messiaen, J. Ongena et al., Nucl. Fusion 39, 1637 (1999).

${ }^{5}$ E. A. Lazarus, J. D. Bell, C. E. Bush et al., Nucl. Fusion 25, 135 (1985).

${ }^{6}$ E. A. Lazarus, J. D. Bell, C. E. Bush et al., J. Nucl. Mater. 121, 61 (1984).

${ }^{7}$ J. Ongena, A. Messiaen, B. Unterberg et al., Plasma Phys. Controlled Fusion 41, A379 (1999).

${ }^{8}$ J. Ongena, R. V. Budny, C. E. Bush et al., Controlled Fusion and Plasma Physics, Proceedings of the 24th European Conference, Berchtesgaden, 1997 (European Physical Society, Geneva, 1997), Part IV, p. 1693.

${ }^{9}$ Proceedings of the First Workshop on Research Programme on Radiative Modes on the Existing European Facilities, Jülich, January 1999 (Institut für Plasmaphysik, Forschungszentrum Jülich, Germany, 1999).

${ }^{10}$ A. Kallenbach, R. Dux, H. S. Bosch et al., Plasma Phys. Controlled Fusion 38, 2097 (1996).

${ }^{11}$ K. Hill, S. D. Scott, M. Bell et al., Phys. Plasmas 6, 877 (1999).

${ }^{12}$ M. Murakami, G. R. McKee, G. L. Jackson et al., 18th IAEA Fusion Energy Conference, Sorrento, 2000 (International Atomic Energy Agency, Vienna, 2000), paper IAEA-CN-77/EX5/1.

${ }^{13}$ H. Kubo, S. Sakurai, N. Asakura et al., in Ref. 12, paper IAEA-CN-77/ EX5/3.

${ }^{14}$ G. Maddison, M. Brix, R. Budny et al., in Ref. 12, paper IAEA-CN-77/ EX5/4.

${ }^{15}$ B. Unterberg, M. Brix, R. Budny et al., in Controlled Fusion and Plasma Physics, Proceedings of the 27th EPS Conference, Budapest 2000, paper P4.038.
${ }^{16}$ G. Jackson, M. Brix, R. Budny et al., in Ref. 15, paper P1.047.

${ }^{17}$ J. Strachan, R. Budny, I. Coffey et al., Plasma Phys. Controlled Fusion 42, A81 (2000).

${ }^{18} \mathrm{U}$. Samm, Proceedings of the 16th IEEE Symposium on Fusion Engineering, 1995 (Institute for Electrical and Electronics Engineers, Piscataway, NJ, 1995), p. 470.

${ }^{19}$ K. H. Dippel, J. Nucl. Mater. 145-147, 3 (1987).

${ }^{20}$ H. R. Koslowski, G. Fuchs, R. Jaspers et al., Nucl. Fusion 40, 821 (2000).

${ }^{21}$ J. Ongena, A. M. Messiaen, U. Samm et al., Plasma Phys. Controlled Fusion 38, 279 (1996)

${ }^{22}$ J. A. Boedo, J. Ongena, R. D. Sydora et al., Nucl. Fusion 40, 209 (2000).

${ }^{23}$ J. Rapp, P. C. DeVries, F. C. Schüller et al., Nucl. Fusion 39, 765 (1999).

${ }^{24}$ M. Tokar', J. Ongena, B. Unterberg, and R. Weynants, Phys. Rev. Lett. 84, 895 (2000).

${ }^{25}$ B. Unterberg, G. Mank, A. Messiaen et al., 18th IAEA Fusion Energy Conference, Sorrento, 2000 (International Atomic Energy Agency, Vienna, 2000), paper IAEA-CN-77/EX5/2.

${ }^{26}$ A. Odblom, P. J. Catto, and S. I. Krasheninnikov, Phys. Plasmas 6, 3239 (1999).

${ }^{27}$ ITER Physics Basis, Nucl. Fusion 39, 2175 (1999).

${ }^{28}$ G. R. McKee, K. Burrell, R. Fonck et al., Phys. Rev. Lett. 84, 1922 (2000)

${ }^{29}$ G. Jackson, M. Murakami, G. R. McKee et al., "Effects of impurity seeding in DIII-D radiating mantle discharges," submitted to Nucl. Fusion.

${ }^{30}$ J. Ongena, M. Evrard, and D. Mccune, Trans. Fusion Technol. 33/2T, 181 (1998).

${ }^{31}$ L. D. Horton, G. D. Conway, N. C. Hawkes et al., Controlled Fusion and Plasma Physics, Proceedings of the 26th EPS Conference, Maastricht, 1999, paper P1.021.

${ }^{32}$ J. Strachan, M. G. Bell, C. E. Bush et al., Nucl. Fusion 39, 919 (1999).

${ }^{33}$ R. D. Sydora, V. K. Decyk, and J. M. Dawson, Plasma Phys. Controlled Fusion 38, 281 (1996)

${ }^{34}$ R. D. Sydora, J-N. Leboeuf, J. M. Dawson et al., 18th IAEA Fusion Energy Conference, Sorrento, 2000 (International Atomic Energy Agency, Vienna, 2000), paper IAEA-CN-77/THP1/27.

${ }^{35}$ C. C. Petty and T. C. Luce, Phys. Plasmas 6, 909 (1999).

${ }^{36}$ Yu. N. Dnestrovskij, S. V. Cherkasov, S. E. Lysenko et al., Nucl. Fusion 38, 373 (1998).

${ }^{37}$ B. Coppi, Comments Plasma Phys. Control. Fusion 5, 264 (1980).

${ }^{38}$ Yu. N. Dnestrovskij, S. V. Cherkasov, S. E. Lysenko, K. N. Tarasyan, J. Ongena, and A. Messiaen, in Ref. 31, paper P2.004.

${ }^{39}$ B. Unterberg, M. Brix, H. G. Esser et al., in Ref. 31, paper 2.035 .

${ }^{40}$ M. Tendler, Plasma Phys. Controlled Fusion 39, 317 (1997). 